\title{
Finding relief at the end of the (Carpal) tunnel: Electrophysiological clues
}

Carpal tunnel syndrome (CTS) is an entrapment mononeuropathy of the median nerve at the wrist as it traverses the anatomically-restricted confines.

The pathophysiology of CTS is usually demyelination of the median nerve due to compression, but axonal loss may coexist in severe cases. Crowding within at the carpal tunnel may be due to multiple causes, including dysthyroid states, arthritis, diffuse increase in the soft tissues, fracture, and infiltrative conditions. However, other less clearly defined mechanisms must also be important, as it is well known that electrophysiological parameters of demyelination and axonal loss do not fully explain all symptomatology.

In some $15 \%$ of CTS, the standard nerve conduction study (NCS) is normal. Yet some reliable method is necessary for the purpose of (i) stratifying the treatment options for early versus delayed surgery, (ii) monitoring axonal loss and, (iii) objectifying post-intervention outcomes. Sometimes the severity of symptoms may be the determining factor in the decision for surgery. As there is no "gold standard" for diagnosing CTS, a combination of electrophysiological parameters may be a reasonable surrogate.

The most sensitive parameter for detecting CTS is the sensory conduction velocity (CV) and distal latency (DL). The motor $\mathrm{CV}$, motor DL and even compound motor action potential (CMAP) will be affected with moderate involvement. The electrophysiological grading proposed by Bland $\mathrm{JD}^{[1]}$ and the American Academy Neuromuscular and Electrodiagnostic Medicine (AANEM) 2011 guidelines $^{[2]}$ is reflective of this gradient of severity. Additional specialized techniques may be used for detecting subtle CTS, for

\begin{tabular}{|l|l|}
\hline \multicolumn{2}{|c|}{ Access this article online } \\
\hline Quick Response Code: & Website: \\
\hline & www.ruralneuropractice.com \\
\cline { 2 - 3 } & \\
\hline & \\
\end{tabular}

example, the second lumbrical- dorsal interosseous latency difference, and combined sensory index (CSI). ${ }^{[3]}$ It is less common to encounter exclusively delayed motor DL or reduced CMAP.

Interventional studies involving carpal tunnel release are heterogeneous and may show improvement and normalization in both motor and sensory parameters, although not necessarily in tandem; complete normality is unusual. ${ }^{[4]}$ In a retrospective review, Malladi et al, ${ }^{[5]}$ found that the improvement in CMAP and motor DL correlated best with resolution of paresthesia but not pain. Sensory DL usually takes a longer time to improve but the patients may feel symptomatic relief within a short time post-operatively. This was hypothesized to be due to reduced spontaneous firing in the irritated nerve than to improvement in conduction block. Padua et $a l_{,}{ }^{[6]}$ found that the pre-surgical electrophysiological grading (based on sensory and motor parameters) could predict the improvements that may be expected post-operatively. Shurr et al, ${ }^{[7]}$ followed post-CTS release hands for up to 12 months and found improvements in both sensory and motor CV at 2 weeks, and sensory and motor DL at 3 months. In another study by Rotman et al, ${ }^{[8]}$ clinical improvement seemed to correlate only with the motor DL, but this result was seen only in patients with motor DL of 4-6 milliseconds and $>6$ milliseconds initially.

Whilst CMAP and motor DL is easily amenable to measurement, one should not ignore the sensory parameters. The sensory component can usually be measured except for the most severe cases (Bland "grade 4" and above, or AANEM grade "severe"). ${ }^{[1,2]}$ Moreover, following the motor parameter alone is not advisable in the mild CTS group where only the sensory parameter is affected predominantly.

Hence, the overriding theme in CTS studies is that not all cases necessarily progresses in the same manner as the severity increases. It is even more challenging in patients who have variant anatomy (Martin-Gruber anomaly) and other co-morbidity (e.g., diabetes mellitus). Documenting a change in a single parameter alone may not be meaningful to the patient. 
In the study presented in this issue by Gupta et al, ${ }^{[9]}$ motor DL improved by $10.3 \%$ in both the mild to moderate CTS groups, but all the sensory parameters showed even larger percentage change from baseline. In the severe to very severe CTS groups, motor DL improved by $9.4 \%$ and CMAP by $35 \%$. Even more spectacular was the sensory parameter becoming recordable in 10/22 hands (i.e., $45.5 \%$ improvement). Perhaps this finding suggests that although monitoring the motor DL is useful, following the sensory parameters were potentially more efficacious. From another perspective and from reasons given in the preceding paragraphs above, one month may be too short for the sensory parameters to show significant normalization. The motor DLs in this study were in general not very prolonged initially, and therefore detecting a small but significant improvement will be difficult.

In conclusion, there will probably not be an ideal trial which reliably tracks a single electrophysiological parameter in tandem with patient's report of symptomatic improvement, simply because the condition is extremely heterogeneous. Just as CTS trials which use symptom reporting as outcome measures may be criticized as lacking objectivity, the neurophysiologic parameters in CTS may improve without the patient experiencing symptomatic relief.

In the authors' opinion, the patient is the final arbiter. The most appropriate treatment (steroids, splint or surgery) is one where there is an improvement in both motor and sensory function, as judged by him or her.
Chun Wai Yip, Yew Long Lo

Department of Neurology, Singapore General Hospital Campus, National Neuroscience Institute, Outram Road, Singapore 169608

Address for correspondence: Dr. Yew Long Lo

Department of Neurology Singapore General Hospital Campus National Neuroscience Institute, Outram Road, Singapore 169608. E-mail: gnrlyl@sgh.com.sg

\section{References}

1. Bland JD. A neurophysiological grading scale for carpal tunnel syndrome. Muscle Nerve 2000;23:1280-3.

2. Robert A Werner, Michael Andary. AANEM Monograph. Electrodiagnostic Evaluation of Carpal Tunnel Syndrome. Muscle Nerve 2011;44:597-607.

3. Robinson LR, Micklesen P, Wang L. Strategies for analyzing nerve conduction data: Superiority of a summary index over single tests. Muscle Nerve 1998;21:1166-71.

4. Prick JJ, Blaauw G, Vredeveld JW, Oosterloo SJ. Results of Carpal tunnel release Eur J Neurol 2003;10:733-6.

5. Malladi N, Mickelsen PJ, Hou J, Robinson LR. Correlation between the combined sensory index and clinical outcomes after carpal tunnel decompression: A retrospective review. Muscle Nerve 2010;41:453-7.

6. Padua L, LoMonaco M, Aulisa L, Tamburrelli F, Valente EM, Padua R, et al. Surgical Prognosis in carpal tunnel syndrome: Usefulness of a preoperative neurophysiological assessment. Acta Neurol Scand 1996;94:343-6.

7. Shurr DG, Blair WF, Bassett G. Electromyographic changes after carpal tunnel release. J Hand Surg Am 1986;11:876-80.

8. Rotman MB, Enkvetchakul BV, Megerian JT, Gozani SN. Time course and predictors of median nerve conduction after carpal tunnel release. J Hand Surg Am 2004;29:367-72.

9. Gupta S, Tewari AK, Nair V, Gupta A. Reliability of motor parameters for follow up after local steroid injection in carpal tunnel syndrome. J Neurosci Rural Pract 2013;4:392-6.

How to cite this article: Yip CW, Lo YL. Finding relief at the end of the (Carpal) tunnel: Electrophysiological clues. J Neurosci Rural Pract 2013;4:377-8.

Source of Support: Nil. Conflict of Interest: None declared.

Announcement

\section{“QUICK RESPONSE CODE” LINK FOR FULL TEXT ARTICLES}

The journal issue has a unique new feature for reaching to the journal's website without typing a single letter. Each article on its first page has a "Quick Response Code". Using any mobile or other hand-held device with camera and GPRS/other internet source, one can reach to the full text of that particular article on the journal's website. Start a QR-code reading software (see list of free applications from http://tinyurl.com/yzlh2tc) and point the camera to the QR-code printed in the journal. It will automatically take you to the HTML full text of that article. One can also use a desktop or laptop with web camera for similar functionality. See http://tinyurl.com/2bw7fn3 or http://tinyurl.com/3ysr3me for the free applications. 